\title{
Study on the biochemistry laboratory performance in notifying critical alerts
}

\author{
J Sowndharya ${ }^{1 *}$, S Aruna $^{2}$, G Jeyachandran ${ }^{3}$ \\ ${ }^{1}$ Tutor, ${ }^{2}$ Assistant Professor, Department of Biochemistry, Government Karur Medical College and Hospital, Karur. \\ ${ }^{3}$ Professor and HOD, Department of Biochemistry, PSG Institute of Medical Sciences and Research, Coimbatore. \\ Email: sowndaryaj27@gmail.com
}

Abstract Background: Critical alert interval is the interval of examination (test) results that indicates an immediate risk to the patient of injury or death. Materials and Methods: All consecutive critical data of the month January 2019 are taken from the critical alert register from a tertiary care hospital/DC/BC/Reg/RR/09-Critical. From LIS, we generated the list of data satisfying the critical alert conditions in the same period January 2019. Results: The distribution had two peaks. The first peak is the tallest i.e.171 alerts are given within 25 minutes. ABG and electrolyte samples processed immediately, contribute to this. This peak is superimposed on a normal distribution with a peak at 50 to 75 minutes interval and a long tail on the right side. Conclusion: Critical alert recording is missed in $26.3 \%$ of total alerts triggered. After correction, $50 \%$ of beta HCG is not recorded in the register. In 171 instances only we have given the alert within 25 minutes.

Key Words: Critical alert, Parameters, Samples, Notification and authorized personnel.

*Address for Correspondence:

Dr. Sowndharya J, Tutor, Department of Biochemistry, Government Karur Medical College and Hospital, Karur

Email: sowndaryaj27@gmail.com

Received Date: 19/10/2019 Revised Date: 10/11/2019 Accepted Date: 24/12/2019

DOI: https://doi.org/10.26611/10021311

\begin{tabular}{|l|l|}
\hline \multicolumn{2}{|c|}{ Access this article online } \\
\hline Quick Response Code: & Website: \\
\hline & www.medpulse.in \\
& \\
\hline
\end{tabular}

\section{INTRODUCTION}

Critical alert interval is the interval of examination (test) results that indicates an immediate risk to the patient of injury or death. When examination results fall within established "alert" or "critical" intervals:

-A physician (or other authorized health professional) is notified immediately.

-Records are maintained of actions taken that document date, time, responsible laboratory staff member, person notified and examination results conveyed, and any difficulties encountered in notifications.

This study is about how effectively it is practiced in the laboratory

\section{MATERIALS AND METHODS}

Institutional human ethics committee approval was obtained. All consecutive critical data of the month January 2019 are taken from the critical alert register from a tertiary care hospital/DC/BC/Reg/RR/09-Critical. From LIS, we generated the list of data satisfying the critical alert conditions in the same period January 2019.

\section{FOLLOW UP}

- We have a LIS based system to trigger, initiate action and record critical alert in $100 \%$ instances after this study.

- We have been working to reduce the time taken for issuing alert and efficiency of tracking the authorized personnel to issue the alert. 
Following are the parameters and their critical alert levels:

\begin{tabular}{cc}
\hline PARAMETERS & CRITICAL ALERT LEVELS \\
\hline Serum amylase & $>200 \mathrm{U} / \mathrm{L}$ \\
Serum ionized calcium & $<0.75$ or $>1.625 \mathrm{mmol} / \mathrm{L}$ \\
Serum total calcium & $<6.0$ or $>13.0 \mathrm{mg} / \mathrm{dL}$ \\
Plasma glucose & $<70$ or $>450 \mathrm{mg} / \mathrm{dL}$ \\
Serum Osmolality & $<240$ or $>320 \mathrm{mOsm} / \mathrm{Kg}$ \\
Blood pH (arterial) & $<7.20$ or $>7.60$ \\
Blood $\mathrm{pO}_{2}$ (arterial) & $<40 \mathrm{~mm} \mathrm{Hg}$ \\
Serum/Plasma sodium & $<120$ or $>160 \mathrm{mEq} / \mathrm{L}$ \\
Serum $/$ plasma potassium & $<3.0$ or $>6.0 \mathrm{mEq} / \mathrm{L}$ \\
Serum $\beta$ HCG & $>1000 \mathrm{mIU} / \mathrm{mL}$ \\
Plasma Troponin T hs & $>15 \mathrm{pg} / \mathrm{mL}$ \\
\hline
\end{tabular}

\section{OBSERVATION AND RESULTS}

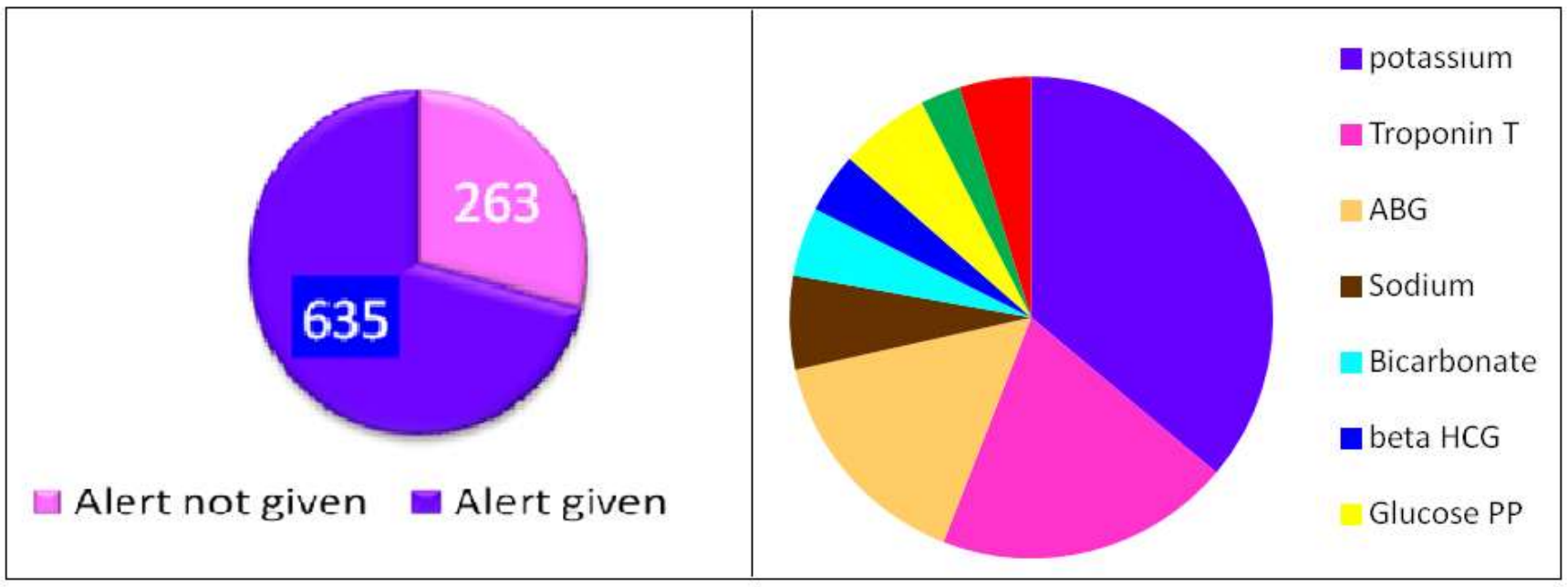

Figure A: Alerts recorded and not recorded

Figure B: Parameter wise alert

\begin{tabular}{cccc}
\multicolumn{4}{c}{ Missed alerts compared with total alerts } \\
\hline & TOTAL ALERTS & NOT RECORDED & \%NOT RECORDED \\
\hline Bilirubin & 3 & 3 & 100 \\
Osmolality & 4 & 3 & 75 \\
Glucose Fasting & 7 & 1 & 14 \\
Calcium & 12 & 9 & 75 \\
Glucose Random & 17 & 4 & 24 \\
Ionised calcium & 24 & 7 & 29 \\
Amylase & 26 & 2 & 8 \\
Glucose PP & 28 & 9 & 32 \\
beta HCG & 36 & 29 & 81 \\
revised & 13 & 6 & 50 \\
Bicarbonate & 41 & 15 & 37 \\
Sodium & 56 & 19 & 34 \\
ABG & 139 & 43 & 31 \\
Troponin T & 177 & 42 & 24 \\
revised & 164 & 29 & 18 \\
Potassium & 322 & 56 & 17 \\
\hline
\end{tabular}




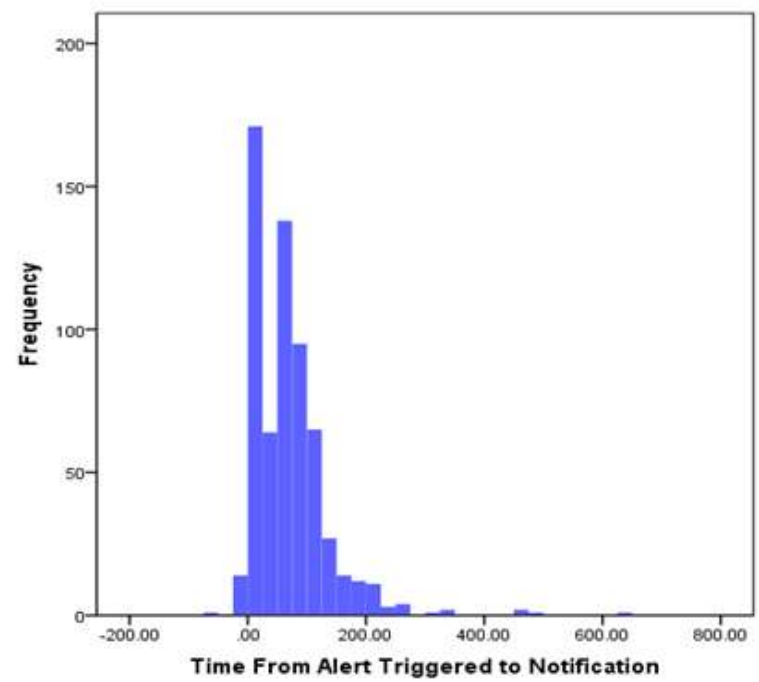

Time duration from alert triggered to alert notification

\section{RESULTS}

The distribution had two peaks. The first peak is the tallest i.e. 171 alerts are given within 25 minutes. ABG and electrolyte samples processed immediately, contribute to this. This peak is superimposed on a normal distribution with a peak at 50 to 75 minutes interval and a long tail on the right side.

\section{CONCLUSION}

Critical alert recording is missed in $26.3 \%$ of total alerts triggered.
After correction, $50 \%$ of beta HCG is not recorded in the register. In 171 instances only we have given the alert within 25 minutes. In 442 instances it took $26 \mathrm{mts}$ to $4 \mathrm{hrs}$. In 12 samples, it took more than 4 hours.

\section{REFERENCES}

1. ISO/IEC 17000, ISO/IEC Guide 2 and ISO/IEC Guide 99

2. ISO 15189- 2012

3. Quality System Procedure Manual $\mathrm{DC} / \mathrm{BC} / \mathrm{QSP} / \mathrm{SOP} / \mathrm{RR} / 11$

Source of Support: None Declared Conflict of Interest: None Declared 